\title{
Imaging faint companions very close to stars
}

\author{
Eugene Serabyn ${ }^{1}$, Dimitri Mawet ${ }^{1}$ and Rick Burruss ${ }^{1}$ \\ ${ }^{1}$ Jet Propulsion Laboratory, California Institute of Technology, Pasadena, CA 91109, USA \\ email: gene.serabyn@jpl.nasa.gov
}

\begin{abstract}
A vortex coronagraph on our extreme adaptive optics "well-corrected subaperture" on the Hale telescope has recently allowed the imaging of the triple-planet HR8799 system with a $1.5 \mathrm{~m}$ subaperture. Moreover, a faint, low-mass companion to a second star was imaged only one diffraction beam width away from the primary. These results illustrate the potential of the vortex coronagraph, which can enable exoplanet imaging and characterization with smaller telescopes than previously thought.
\end{abstract}

Keywords. instrumentation: adaptive optics, planetary systems, binaries (including multiple): close

\section{Vortex Coronagraphy}

The vortex coronagraph provides close to ideal small-angle performance (Guyon et al. 2006), allowing observations very close to stars. This small-angle capability allows a significant reduction in the size of potential space telescopes aimed at detecting and characterizing exoplanets. Over the past several years, we have thus implemented a highcontrast vector vortex coronagraph on the $1.5 \mathrm{~m}$ well-corrected subaperture of the Palomar Hale telescope, in order to begin to demonstrate its capabilities (Mawet et al. 2010). Indeed, even with our rather small subaperture, the observational results to date have been competitive with results obtained at much larger telescopes.

Our well-corrected subaperture had earlier already enabled observations of brown dwarfs and debris disks, using a four-quadrant phase mask coronagraph. With the upgrade to vector vortex masks, and the improvement of our contrast performance to roughly $10^{-5}$ within $\sim \lambda / \mathrm{D}$ of the star (Burruss et al. 2010), we have been able to image the triple planet HR8799 system with our small aperture coronagraph (Figure 1; Serabyn et al. 2010). Recently, we have also imaged a binary star's faint secondary companion only $\sim 1 \lambda / \mathrm{D}$ from the primary (Figure 2; Mawet et al. in prep.). Thus, both the vortex's small-angle performance, and the utility of small telescopes for exoplanet imaging have begun to be validated. Indeed, a well-corrected subaperture employing a coronagraph capable of reaching small angles is a very promising method for quickly initiating exoplanet observations with a given telescope, especially important as we head toward the era of 30-40 m telescopes, where a well-corrected subaperture exceeding 10 $\mathrm{m}$ in diameter can quickly provide state-of-the-art high-contrast observations.

\section{References}

Burruss, R. S., Serabyn, E., Mawet, D. P., Roberts, J. E., Hickey, J. P., Rykoski, K., Bikkannavar, S., \& Crepp, J. R. 2003, Proc. SPIE, 7736, 77365X

Guyon, O., Pluzhnik, E. A., Kuchner, M. J., Collins, B., \& Ridgway S. T. 2006, ApJS, 167, 81

Mawet, D., Serabyn, E., Liewer, K., Burruss, R., Hickey, J., \& Shemo, D. 2010, ApJ 709, 53

Serabyn, E., Mawet, D., \& Burruss, R. 2010, Nature, 464, 1018 


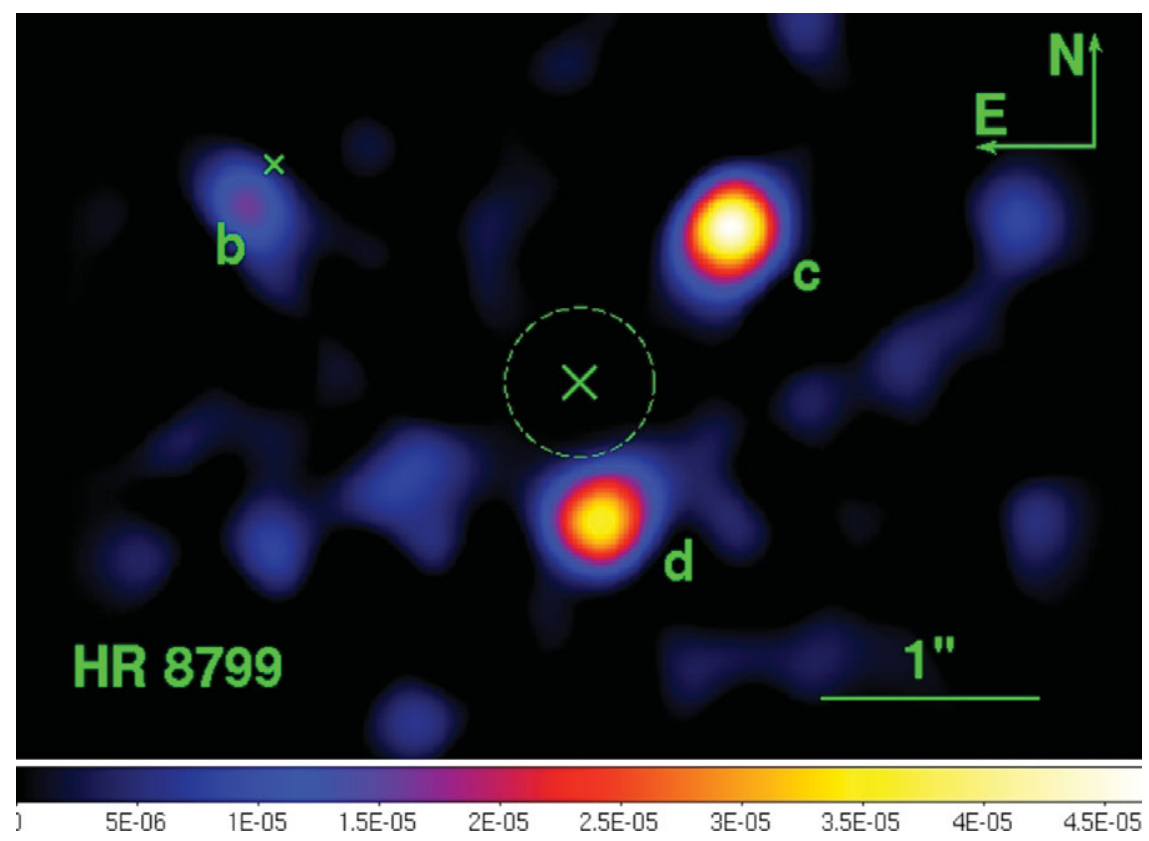

Figure 1. The HR8799 exoplanets imaged with our $1.5 \mathrm{~m}$ well-corrected subaperture.

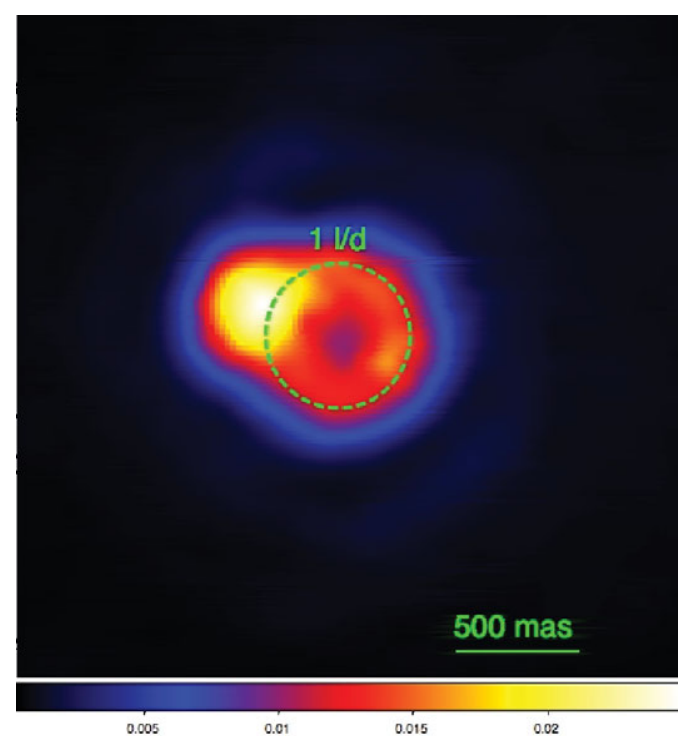

Figure 2. A low-mass binary companion seen at $\sim \lambda / \mathrm{D}$ with our vortex coronagraph. The residuals of the primary star appear as a faint ring 